\title{
Comparison of Plasma Dynamics in Plasma Focus Devices PF1000 and PF400
}

\author{
Amir Shakya*, Prakash Gautam and Raju Khanal \\ Central Department of Physics, Tribhuvan University, Kirtipur, Kathmandu, Nepal \\ *Correspondence to:shakyaamir12@gmail.com
}

\begin{abstract}
In this work we have simulated two different sizes of plasma focus devices: PF400, a small sub-kJ plasma focus device in operation at the Atomic Energy Commissionin Chile and PF1000, a 1 MJ deviceat the International Centre for Dense Magnetised Plasmas (ICDMP) in Warsaw, Poland. Our aim is to compare the plasma dynamics observed in two different sizes of the devices. We compare the experimentally obtained data with the data predicted from our numerical experiments using the Lee model code. It has been observed that the peak current of the total current waveform, pinch current calculated from total current, and neutron yield agreereasonably well with published experimental data, however, slight differences are found in other parameters.
\end{abstract}

Keywords: Plasma focus, Lee model code, Total current waveform, Peak current, Pinch current, Neutron yield

\section{Introduction}

A dense plasma focus (DPF) is a machine that produces short-lived plasma that is hot and dense enough to cause nuclear fusion and the emission of X-rays and neutrons. It was invented in the early 1960's by Filippov et al. [1962] and independently by Mather [1964]. In this device, the magnetic energy is stored behind the moving current sheath. A portion of this energy is converted into plasma energy during the rapid collapse (Z-pinch) of the current sheath towards the axis beyond the end of central electrode. A simple capacitor discharge is sufficient to power the plasma focus. Thus, a DPF is a compact powerful pulsed source of multi radiation. The plasma focus is similar to the high-intensity plasma gun, which ejects plasma in the form of a plasmoid, without pinching it. DPF finds its application in microelectronics lithography, surface micromachining, pulsed X-ray and neutron source for medical and security inspection applications and materials modification, among others [Lee et al., 2008 and Lee, 2014].

The working of a DPF can be described in five phases, namely, the axial phase, radial inward shock phase, radial reflected shock phase, slow compression or pinch phase and expanded column phase. Based on this a numerical code, called the Lee Model Code, has been developed for studying various DPF devices and is tested over the past 3 decades [Lee, 2014]. Lee code couples the electrical circuits with plasma focus dynamics, thermodynamics and radiation, enabling realistic simulation of all gross focus properties [Lee, 1984, Lee, 2015, Lee and Saw, 2008, Lee et al., 2008]. It has been used in design and interpretation of Mather type plasma focus experiments and as a complementary facility to provide diagnostic references in different gases. The code was first described in 1983 and used 
in the design and interpretation of experiments, and recently it is also being used to produce reference numbers for deuteron beam number and scaling trends for these with PF storage. These beam properties has been extended to all gases. The code has been used extensively in several machines including UNU/ICTP PFF, NX2 and NX1 and adapted for the Filippovtype plasma focus DENA as well [Lee, 2014]. The Lee Model code uses four parameters, two axial mass and current factors and two radial mass and current factors to fit a computed current trace to the measured current trace of any plasma focus [Lee, 2015, Lee, 2014, and Saw et al., 2015]. Once the computed current waveform is fitted to the measured value, the configured plasma focus is equivalent energywise, charge-wise, momentum-wise and masswise to the actual plasma focus; all these processes having been taken care of by the adjustment of the four parameters and the code which models the dynamics, electrodynamics, thermodynamics and radiation interactively. From the fitted current waveform, it has been found that the dynamics, thermodynamics, soft $\mathrm{X}$-rays, fusion neutrons (in D and $\mathrm{D}-\mathrm{T}$ ) and ion and plasma properties in various gases are consistent with observed values.

In this work we compare different extensive properties of PF1000 and PF400, after fitting computed current waveform and measured current waveform obtained from published papers. The data for this paper has been collected and combined with new numerical experiments using the Lee code.

\section{Procedure for the Numerical Experiments}

The Lee model code is first configured to work as a plasma focus by imputing the tube parameters: cathode radius (b), anode radius (a) and anode length $\left(\mathrm{z}_{0}\right)$ together with the bank parameters:static inductance $\left(\mathrm{L}_{0}\right)$,capacitance $\left(\mathrm{C}_{0}\right)$ and stray circuit resistance $\mathrm{r}_{0}$ and operational parameters:voltage $\left(\mathrm{V}_{0}\right)$, pressure $\left(\mathrm{P}_{0}\right)$ and the fill gas. The method of configuring the Lee model code is discussed in great detail by Gautam and Khanal [2015]. The standard practice is to fit the computed total discharge current waveform using four model parameters representing the mass swept-up factor fm, the plasma current factor fc for the axial phase, radial phase mass and current factors fmr and fcr for the radial phases. It is known that current trace of the focus is one of the best indicators of gross performance. The axial and radial phase dynamics and the crucial energy transfer into the focus pinch are among the important information that is quickly apparent from the current trace. This explains the importance of current fitting [Lee, 2015].

\section{Results}

The results obtained by the simulation of the two PF devices (PF1000 and PF400) are first presented separately and are then compared.

\subsection{PF1000}

The experimentally measured current trace is taken from [Gribkov et al., 2007] and is operated with voltage $27 \mathrm{kV}$ and pressure 3.5 Torr. Numerically computed current waveform is fitted to the measured current waveform and the fit is found reasonably wellup to the end of the radial pinch phase but no attempt has been made to fit beyond end of radial phase. We found fine tunning wave form for the following values.

Bank parameters:

$\mathrm{L}_{0}=33.5 \mathrm{nH}, \mathrm{C}_{0}=1,332 \mu \mathrm{F}, \mathrm{r}_{0}=6.1 \mathrm{~m} \Omega$ 
Tube parameters:

$$
\mathrm{b}=16 \mathrm{~cm}, \mathrm{a}=11.5 \mathrm{~cm}, \mathrm{z}_{0}=60 \mathrm{~cm}
$$

Operating parameters:

$$
\mathrm{V}_{0}=27 \mathrm{kV}, \mathrm{P}_{0}=3.5 \text { Torr deuterium }
$$

Together with the following fitted model parameters:

$$
\mathrm{f}_{\mathrm{m}}=0.13, \mathrm{f}_{\mathrm{c}}=0.7, \mathrm{f}_{\mathrm{mr}}=0.35 \text { and } \mathrm{f}_{\mathrm{cr}}=0.65
$$

Figure 1 shows the comparison between computed Itotalobtained by using above parameters with the measured Itotal [Gribkov et al., 2007]. As the fitting shows very good matching the computed dynamics, currents, and other properties of these plasma focus discharges were deemed to be correctly simulated.

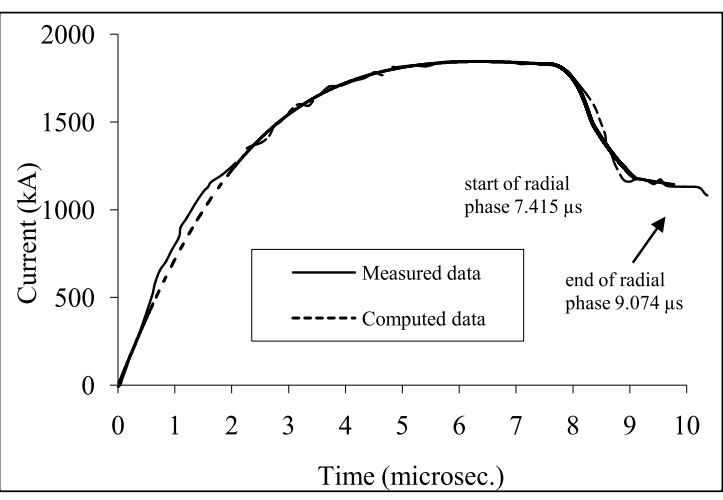

Figure 1. A typical fit of a low inductance PF1000; showing reasonable agreement between computed and measured waveforms

From the computed current curve we observed that the radial phase starts at time $7.415 \mu \mathrm{s}$ and end of the radial phase is at time $9.074 \mu \mathrm{s}$, we note that the agreement of the computed curve with the measured curve up to this point is fair. The start of the pinch phase was obtained as $9.34 \mu \mathrm{s}$. At this time Ipinchis computed as $827 \mathrm{kA}$.

\section{2 $\quad$ PF400}

The experimental data ofcurrent curve at $28 \mathrm{kV}$ and 6.6 Torrfor the PF400 is taken from the work by Lee et al. [2009]. As usual we first fit the current wave form, for finding the model parameters fm, fc, fmr and fcras discussed in the case of PF1000. To obtain the reasonably good fit the following bank and tube parameters $\left(\mathrm{L}_{0}, \mathrm{C}_{0}\right.$ and $\mathrm{z}_{0}$ refitted and $\mathrm{r}_{0}$ fitted $)$ are used:

Bank parameters:

$\mathrm{L}_{0}=38 \mathrm{nH}, \mathrm{C}_{0}=0.88 \mu \mathrm{F}, \mathrm{r}_{0}=10 \mathrm{~m} \Omega$

Tube parameters:

$\mathrm{b}=1.55 \mathrm{~cm}, \mathrm{a}=0.6 \mathrm{~cm}, \mathrm{z}_{0}=1.7 \mathrm{~cm}$

Operating parameters:

$\mathrm{V}_{0}=28 \mathrm{kV}, \mathrm{P}_{0}=6.6$ Torr Deuterium

Together with the following fitted model parameters:

$\mathrm{f}_{\mathrm{m}}=0.08, \mathrm{f}_{\mathrm{c}}=0.7, \mathrm{f}_{\mathrm{mr}}=0.11$ and $\mathrm{f}_{\mathrm{cr}}=0.7$

The computed current waveform fitted to the measured current waveform [Lee et al. 2009] is shown in Figure 2. The fit is good up to the end of the radial pinch phase. No attempt has been made to fit beyond end of radial phase.

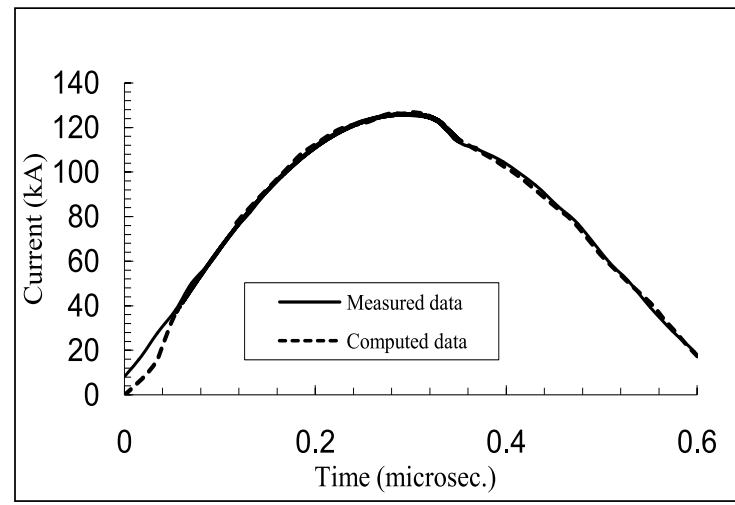

Figure 2. A typical fit of a low inductance PF400; showing reasonable agreement between computed and measured waveforms 
From the computed current curve, we observed that the radial phase starts at time $0.308 \mu$ s and end of the radial phase is at time $0.348 \mu$ s, we note that the agreement of the computed curve with the measured curve up to this point is fair. The start of the pinch phase was obtained as 0.35 $\mu \mathrm{s}$. At this time Ipinchis computed to be $81 \mathrm{kA}$ with minimum radius of $0.09 \mathrm{~cm}$, the peak value of current (I peak) is $126 \mathrm{kA}$ at time $0.32 \mu \mathrm{s}$.

\subsection{Comparing the parameters for a large PF (PF1000) with a small PF (PF400)}

After fitting the computed current waveform with measured current waveforms of PF1000 and PF400 the computed parameters are listed in Table 1.

Table 1: Comparison of various parameters obtained by the simulation of a small (PF400) and a large (PF1000) PF device

\begin{tabular}{|c|c|c|c|}
\hline$\underline{\text { Parameter }}$ & $\frac{\text { PF1000 }}{\left(27 \mathrm{kV} 3.5 \text { Torr } \mathrm{D}_{2}\right)}$ & 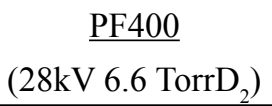 & $\begin{array}{c}\underline{\text { Ratio }} \\
(\mathrm{PF} 1000 / \mathrm{PF} 400)\end{array}$ \\
\hline Stored Energy $\mathrm{E}_{\mathrm{o}}$ in $\mathrm{kJ}$ & 485.51 & 0.37 & 1312.2 \\
\hline Pressure in Torr, $\mathrm{P}_{\mathrm{o}}$ & 3.5 & 6.6 & 0.5 \\
\hline Anode radius a in $\mathrm{cm}$ & 11.6 & 0.6 & 19.3 \\
\hline $\mathrm{c}=\mathrm{b} / \mathrm{a}$ & 1.39 & 2.6 & 0.5 \\
\hline anode length $z_{\mathrm{o}}$ in $\mathrm{cm}$ & 60 & 1.7 & 35.3 \\
\hline final pinch radius $r_{\min }$ in $\mathrm{cm}$ & 2.19 & 0.086 & 25.5 \\
\hline pinch length $\mathrm{z}_{\max }$ in $\mathrm{cm}$ & 18.78 & 0.87 & 21.6 \\
\hline pinch duration in ns & 265.76 & 6.15 & 43.2 \\
\hline $\mathrm{r}_{\min } / \mathrm{a}$ & 0.2 & 0.145 & 1.4 \\
\hline $\mathrm{z}_{\max } / \mathrm{a}$ & 1.63 & 1.46 & 1.1 \\
\hline $\mathrm{I}_{\text {peak }}$ in $\mathrm{kA}$ & 1844.71 & 125.88 & 14.7 \\
\hline $\mathrm{I}_{\text {peak }} / \mathrm{a}$ in $\mathrm{kA} / \mathrm{cm}$ & 159.72 & 209.8 & 0.8 \\
\hline $\mathrm{S}=\left(\mathrm{I}_{\text {peak }} / \mathrm{a}\right) /\left(\mathrm{P}_{\mathrm{o}}{ }^{1 / 2}\right)(\mathrm{kA} / \mathrm{cm}) / \operatorname{Torr}^{1 / 2}$ & 85.4 & 81.7 & 1.0 \\
\hline $\mathrm{I}_{\text {pinch }}$ in $\mathrm{kA}$ & 826.73 & 80.99 & 10.2 \\
\hline $\mathrm{I}_{\text {pinch }} / \mathrm{I}_{\text {peak }}$ & 0.448 & 0.64 & 0.7 \\
\hline Peak induced voltage in $\mathrm{kV}$ & 40.134 & 14.17 & 2.8 \\
\hline peak axial speed in $\mathrm{cm} / \mu \mathrm{s}$ & 11.2 & 9 & 1.2 \\
\hline peak radial shock speed $\mathrm{cm} / \mu \mathrm{s}$ & 16.4 & 29.28 & 0.6 \\
\hline peak radial piston speed $\mathrm{cm} / \mu \mathrm{s}$ & 12.2 & 19.58 & 0.6 \\
\hline peak temperature in $10^{6} \mathrm{~K}$ & 0.952 & 4.4 & 0.2 \\
\hline neutron yield $\mathrm{Y}_{\mathrm{n}}$ in $10^{6}$ & $1.1 \times 10^{5}$ & 1.05 & 104761.9 \\
\hline Measured $Y_{n}$ in $10^{6}:$ range & $(2-7) \times 10^{4}$ & $0.9-1.2$ & \\
\hline
\end{tabular}




\section{Conclusion}

In this work we simulated two different plasma focus devices, namely a large one (PF1000) and a small one (PF400). The current trace was compared with the experimentally measured current waveform of the respective devices for given operational parameters. For both of the devices we obtained a good fit up to the end of radial phases; giving confidence that all the computed results including trajectories and speeds, densities, temperatures and neutron yields are a fair simulation of the actual PF1000 and PF400 experiments. The speed factor $\mathrm{S}$ for both of the devices is nearly the same. This value signifies that all plasma focus devices, big and small, operate with essentially the same energy per unit mass when optimized for neutron yield. It is interesting to note that the radial shock speed in case of PF400 $(29.28 \mathrm{~cm} / \mu \mathrm{s})$ is much more than for the larger device PF1000 (16.4 $\mathrm{cm} / \mu \mathrm{s})$. This implies that higher temperatures can be achieved in smaller devices relatively easily. The comparison presented here between a large and a small plasma focus devices yields various interesting parameters to be considered before conducting real experiments.

\section{Acknowledgments}

Editor N. P. Chapagain thanks the reviewers for their assistance in evaluating this paper

\section{References}

Filippov, N. V., Filippova T. I., and Vinogradov, V. P. (1962), Dense high temperature plasma in a noncylindrical Z-pinch compression, Nucl. Fusion Suppl. 2, 577 - 587.

Gautam, P. and Khanal, R. (2015), Introduction to numerical experiment on plasma focus using Lee Model Code, The Himalayan Physics 5, 136 - 140.
Gribkov, V. A. et al. (2007), Plasma dynamics in the PF-1000 device under full-scale energy storage: II. Fast electron and ion characteristics versus neutron emission parameters and gun optimization perspectives, J. Phys. D: Appl. Phys. 40, 3592 - 3607, doi:10.1088/00223727/40/12/008.

Lee, S. (1984), Radiations in plasmas, vol. II, ed. by B.E. McNamara, World Scientific, Singapore, $978-987$.

Lee, S. (2014), Plasma focus radiative model: review of the Lee model code, J. Fusion Energ. 33, 319 -335, DOI 10.1007/s10894-014-9683-8.

Lee, S. (2015), Radiative dense plasma focus computation package: http://www.plasmafocus. net; http://www.intimal.edu.my/school/fas/ UFLF/ (archival websites)

Lee, S., and Lee, P, Saw, S. H., and Rawat, R. S. (2008), Numerical experiments on plasma focus pinch current limitation, Plasma Phys. Control. Fusion 50(6), 065012, doi:10.1088/0741$3335 / 50 / 6 / 065012$.

Lee, S. and Saw, S. H. (2008), Pinch current limitation effect in plasma focus, Appl. Phys. Lett. 92(2), 021503, DOI: 10.1063/1.2827579

Lee, S., Saw, S. H., Soto, L., Springham, S. V., Moo, S. P. (2009), Numerical experiments on plasma focus neutron yield versus pressure compared with laboratory experiments, Plasma Phys. Control. Fusion 51, 075006, doi: 10.1088/07413335/51/7/075006.

Mather, J. W. (1964), Investigation of the high energy acceleration mode in the coaxial gun, Phys. Fluids Suppl. 7, 28 - 34

Saw, S. H. et al. (2015), Comparison of measured neutron yield versus pressure curves for FMPF3, NX2 and NX3 plasma focus machines against computed results using the Lee model code, $J$. Fusion Energ. 34, 474 - 479, DOI 10.1007/ s10894-014-9824-0. 